\title{
A Hierarchical Reliability Control Method for a Space Manipulator Based on the Strategy of Autonomous Decision-Making
}

\author{
Xin Gao, Yifan Wang, Hanxu Sun, Qingxuan Jia, Gang Chen, Mingtao Du, and Yukun Yang \\ School of Automation, Beijing University of Posts and Telecommunications, No. 10, Xitucheng Road, Haidian District, \\ Beijing 100876, China \\ Correspondence should be addressed to Xin Gao; xlhhh74@bupt.edu.cn \\ Received 16 February 2016; Revised 13 May 2016; Accepted 15 June 2016 \\ Academic Editor: Hamid M. Lankarani \\ Copyright (C) 2016 Xin Gao et al. This is an open access article distributed under the Creative Commons Attribution License, which \\ permits unrestricted use, distribution, and reproduction in any medium, provided the original work is properly cited.

\begin{abstract}
In order to maintain and enhance the operational reliability of a robotic manipulator deployed in space, an operational reliability system control method is presented in this paper. First, a method to divide factors affecting the operational reliability is proposed, which divides the operational reliability factors into task-related factors and cost-related factors. Then the models describing the relationships between the two kinds of factors and control variables are established. Based on this, a multivariable and multiconstraint optimization model is constructed. Second, a hierarchical system control model which incorporates the operational reliability factors is constructed. The control process of the space manipulator is divided into three layers: task planning, path planning, and motion control. Operational reliability related performance parameters are measured and used as the system's feedback. Taking the factors affecting the operational reliability into consideration, the system can autonomously decide which control layer of the system should be optimized and how to optimize it using a control level adjustment decision module. The operational reliability factors affect these three control levels in the form of control variable constraints. Simulation results demonstrate that the proposed method can achieve a greater probability of meeting the task accuracy requirements, while extending the expected lifetime of the space manipulator.
\end{abstract}

\section{Introduction}

With the development of space technology and the increasing of the aerospace application tasks, robotic manipulators are playing an increasingly important and diverse role in space and aerospace applications [1-3]. The overall development trends of the spacecrafts show increasing expense and long life characteristics. The function of the space mechanisms is increasing, and their structure is becoming more complex [4-8]. Consequently with the growth of the working hours in orbit, the space mechanism is bound to have many unpredictable new failure modes exposed. Meanwhile, most of the space mechanisms are deployed in extravehicular roles making it difficult to maintain them. Therefore, it is necessary to achieve high reliability and long life for space mechanisms to complete China's manned spaceflight, deep space exploration, and other major national special tasks.
Furthermore it is also one of the main difficulties of advancing the world's aerospace enterprises [9-12].

According to analysis of a large number of literature researches, more than 156 on-orbit faults of 129 aircrafts happened from 1980 to 2005. According to the fault causes, the electrical/electronic failure accounted for $45 \%$, mechanical failure accounted for $32 \%$, software failure accounted for $6 \%$, and the failure which cannot locate accurately accounted for $17 \%$. Electrical/electronic failure and software failure mostly can be repaired in orbit, but $80 \%$ of mechanical failures led to whole failure of the task. It occurs often that space mechanisms' service life is shortened due to improper operation strategy.

In practice, the reliability of the product can be divided into the inherent reliability and the operational reliability [13]. The inherent reliability of the product is established during the process of the product design and manufacturing 
and can be controlled by the product developers [14]. The operational reliability is a characteristic that exhibits the product's ability to maintain performance in actual use. In addition to its inherent reliability factors, we should also consider the impact of product installation, operation, maintenance, security, and other factors $[15,16]$. Therefore, enhancing the system reliability of the space mechanisms requires consideration of both the inherent reliability and the operational reliability, and particular attention must be paid to the operational reliability.

System control theory is the integrated optimization theory of the control system, which is developed on the basis of the ideas and working methods of systems theory and cybernetics. By analyzing the internal relations of components in the complex systems and considering the relationship among the various parts of the system, on the basis of the control theory, it optimizes and adjusts the process control of the systems to achieve optimal overall system status $[17,18]$.

There are very diverse types of space mechanisms and some are not controllable, such as trusses and other similar support structures [19]. Some can be controlled, such as robotic space manipulator and navigation satellite link drive mechanisms [20, 21]. For the controllable space mechanisms (such as manipulators) containing multiple moving parts, the reliability can be improved by adjusting the control strategy, optimizing the control parameters during the service period, which can extend the working life of the robot arm and reduce the use and maintenance costs of the manipulator.

Existing control methods, such as PID control, variable structure control, fuzzy control, neural network control, and predictive control, have been widely used as the control technology of space mechanisms [22-31]. Existing control models are merely concerned about the impact of factors associated with the effect of task execution, with little consideration about the operational reliability factors associated with the working life of space mechanisms [32-35]. Reference [36] considers the operational reliability for the space manipulator, but it constructed the operational reliability system control model based on the degree of operational reliability, which comes from the perspective of probability distribution. These methods mentioned above do not take into account the factors related to the effect of task execution and the operational reliability factors associated with the life from the system level simultaneously. This leads to shortening the service period of space mechanisms.

Therefore, it is necessary to study and develop an operational reliability system control method for space robotic mechanisms. The approach is to (1) determine the mapping relationship between the reliability factors and control variables and (2) construct the system control model of operational reliability for the space mechanisms, by active regulation of the control variables with three objectives: (i) to minimize the cost when it performs a task under normal condition, (ii) to maximize the mission success rate when it is under abnormal condition, and (iii) to minimize performance degradation during its service years. By constructing a multiobjective optimization function set including tasks reliability, dynamic geometry physical parameters, and time-varying dynamic characteristics, we are going to (1) study the related methods of real-time adjustment and control to reliability of space mechanism by utilizing the optimization function set and (2) construct the fundamental theoretical framework of operational reliability system control of aerospace mechanisms. This will provide the basic theory and an effective means of implementation to ensure satisfying the long life and the high reliability requirements of the space mechanisms during the period of its service in orbit. This is a multidisciplinary research effort based upon multiple control-related fields.

This will be accomplished as follows. First, a division method of factors affecting the operational reliability is proposed, which divides the operational reliability factors into task-related factors and cost-related factors. Then the mapping relationships between the two kinds of factors and control variables are established. Based on this, an optimization model which integrates multiple variables and multiple constraints is constructed. Second, a hierarchical system control model which introduces the operational reliability factors is constructed. According to the model, the control of the space manipulator is divided into three layers: a task planning layer, a path planning layer, and a motion control layer. Operational reliability related performance parameters are measured as the system's feedback. Taking the factors affecting the operational reliability into consideration, the system can autonomously decide which control layer of the system should be optimized and how to optimize it using a control layer adjustment decision module. The operational reliability factors affect these three control layers in the form of constraints on control variables. In the task planning layer and path planning layer, the plan is adjusted by changing the constraints on the control variables and optimization criteria. In the motion control layer, performance is improved by modifying the controller parameters.

Meanwhile, an abnormality diagnostic module analyzes data from a sensor monitoring module to determine whether there is a fault and, if there is a fault, the location of the joint with the fault and, within the joint, the source of the fault, such as a failed actuator and sensor. After a fault is detected and its location is identified, the degree of performance degradation is determined by an operational capacity influence function.

If the fault results in joint performance degradation, as opposed to complete joint failure, motion performance constraints and optimization criteria are adjusted, and noise suppression measures are performed. The adjustment amounts to a correction of the motion control will ensure maximizing probability of task completion under the fault condition.

If the fault is a complete joint failure, a fault self-treatment is implemented. This will allow the manipulator to try to complete its tasks. To do this, the control model is adjusted and reconstructed. The failed joint may cause rapid changes in the parameters in the control models, and these rapid changes must be suppressed to avoid possible damage to the manipulator. After the control model reconstruction and online adjustment are performed, the feasibility of task stage division is redetermined. If it is not feasible, the task constraints and optimization criteria are updated and the task 


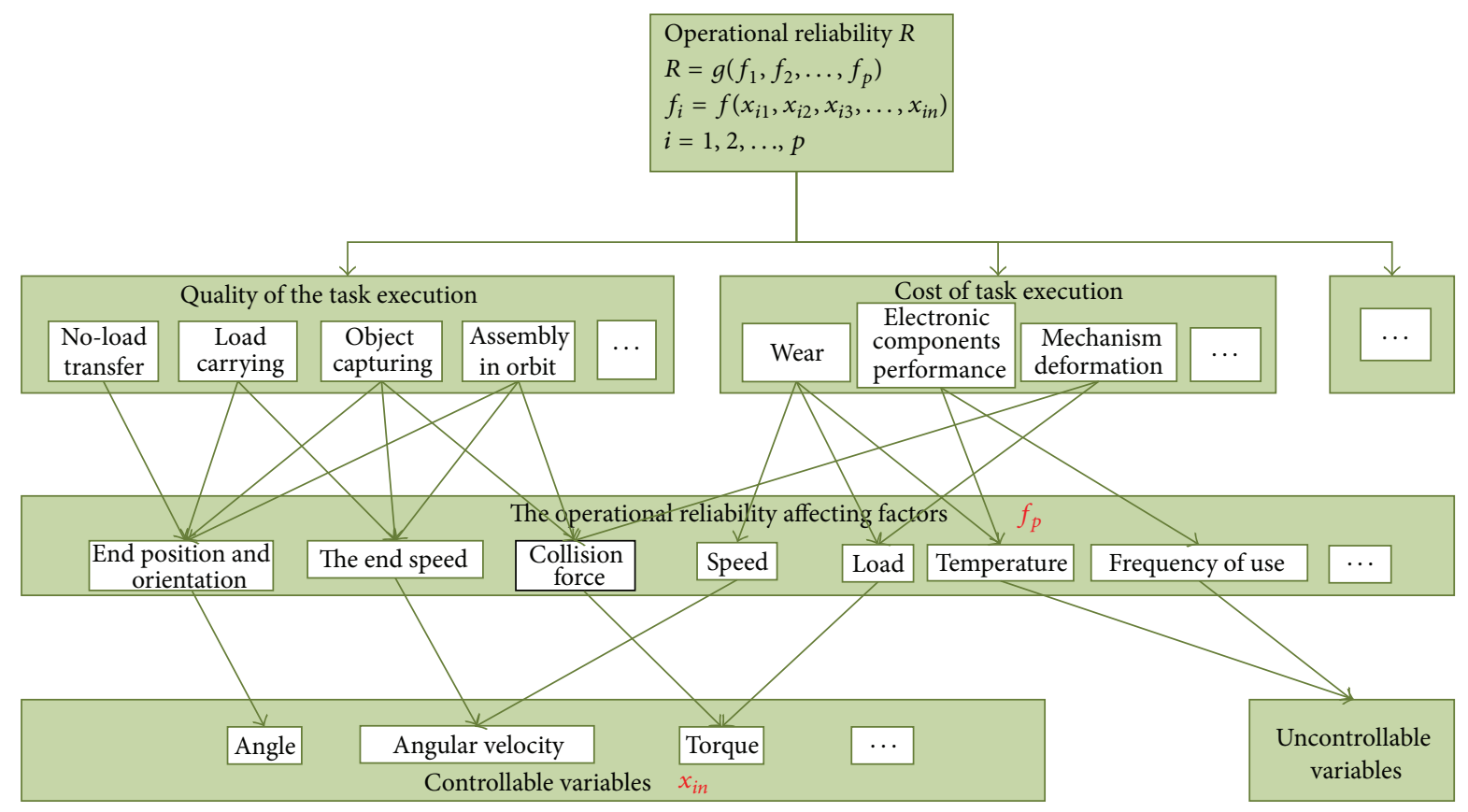

FigURE 1: Multiconstrained multivariable integrated optimization model.

is replanned. This is the subject of parallel research efforts, so the details are not presented in this paper.

\section{The Multiconstrained Multivariable Integrated Optimization Model of the Operational Reliability}

According to reliability theory, the operational reliability factors of the space manipulator are identified. Then we divide the factors into task-related factors and cost-related factors. Necessary but not sufficient conditions for the system's high operational reliability are high quality and low cost of the task execution.

Based on the determined reliability factors, a mathematical model of each factor is established and the model variables are determined; then the model variables are divided into controllable variables and uncontrollable variables. The mapping relationships between the operational reliability factors and controllable variables are established according to the mathematical model of each factor and the division of control variables.

Based on the above analysis, a multiconstrained multivariable integrated optimization model is built in order to improve the operational reliability, as shown in Figure 1. The method presented subsequently is used to improve the operational reliability of space manipulator by adjusting the control variables in the control process, thereby to improve the success rate of the tasks implementation and extend the life of the equipment.

In Figure 1, the operational reliability $R$ of space manipulator is determined by a series of operational reliability factors $f_{1}, f_{2}, \ldots, f_{p}$. Each kind of factor is affected by a number of variables $x_{i 1}, x_{i 2}, x_{i 3}, \ldots, x_{i n}$. The factors are divided into task-related factors and cost-related factors.
Based on the task requirements of space manipulator on orbit, the key indicators related to the typical task success or not are analyzed from the perspective of the task completion and the quality of task completion. Then the task-related factors are determined.

(1) No-Load Transfer Task. The no-load transfer task describes a transient motion between two configurations of space manipulator under no-load conditions. The main factors affecting the task completion and the quality of task completion are the end positioning and orientation, end speed, base attitude, base speed, flexible vibration, and so forth.

(2) Handling Task. The handling task describes a transient motion between two configurations of space manipulator with load, such as space lab transferring, satellite releasing, and assisting the astronaut extravehicular activity. The main factors affecting the task completion and the quality of task completion are base disturbing force, base attitude, base speed, end operating force, the end positioning and attitude and end speed, and so forth.

(3) Capturing Task. The capturing task means that the end of the robotic arm directly captures or dockings the payload target. The main factors affecting the task completion and the quality of task completion are the accuracy of end positioning and attitude, end speed, the collision force and base disturbing force, and so forth.

For the cost-related factors, first, the task-specific costs are classified based on the characteristics of the space manipulator and engineering experiences, into categories such as wear, performance degradation, failure of electronic components, and structural deformation. Then, based on the 
classification, affecting each execution, cost factors are analyzed, such as the effects of the load, speed, and temperature on wear, the effects of power, temperature, and frequency of use on the electronic component performance degradation, and the effects of collision and overloading on the mechanism deformation.

Furthermore, with some influence factors of operational reliability as examples, the relationships between them and control variables are shown as follows.

2.1. End Position and Orientation. The transformation ${ }_{i}^{i-1} T$, which transforms coordinate system $\{i-1\}$ into coordinate system $\{i\}$, can be considered as the product of the following four subtransformations: $Z_{i-1}$ rotates about $X_{i-1}$ by $\alpha_{i-1} ; Z_{i-1}$ translates along $X_{i-1}$ by $a_{i-1} ; X_{i-1}$ rotates about $Z_{i}$ by $\theta ; X_{i-1}$ translates along $Z_{i}$ by $d_{i}$ [37].

Using the sequence of joint angle $q=\left\{q_{1}, q_{2}, \ldots, q_{n-1}, q_{n}\right\}$, according to the transformation relationship between Denavit-Hartenberg parameters and coordinate system, the general expression of link transformation matrix ${ }_{i}^{i-1} T$ can be obtained:

$$
{ }_{i}^{i-1} T=\left[\begin{array}{cccc}
c q_{i} & -s q_{i} & 0 & a_{i-1} \\
s q_{i} c \alpha_{i-1} & c q_{i} c \alpha_{i-1} & -s \alpha_{i-1} & -d_{i} s \alpha_{i-1} \\
s q_{i} s \alpha_{i-1} & c q_{i} s \alpha_{i-1} & c \alpha_{i-1} & d_{i} c \alpha_{i-1} \\
0 & 0 & 0 & 1
\end{array}\right]
$$

Then, the link transformations can be multiplied together to find the single transformation that relates frame $\{N\}$ to frame $\{0\}$ : the linear transformation from frame $\{n\}$ to frame $\{0\}$ can be obtained by multiplying each transformation matrix in order:

$$
{ }_{n}^{0} T={ }_{1}^{0} T{ }_{2}^{1} T{ }_{3}^{2} T \cdot{ }_{n}^{n-1} T .
$$

${ }_{n}^{0} T$ is usually called the transformation matrix of manipulator. If position vector $P$ and rotation matrix $R=\left[\begin{array}{lll}n & o & a\end{array}\right]$ represent position and orientation of the end effector, the manipulator kinematics equation can be defined as follows:

$$
\left(\begin{array}{cccc}
{ }_{n}^{0} R & & { }^{0} P \\
0 & 0 & 0 & 1
\end{array}\right)={ }_{1}^{0} T{ }_{2}^{1} T{ }_{3}^{2} T \cdots{ }_{n}^{n-1} T .
$$

The relationship between position and orientation of the end effector and the joint angle is established.

2.2. Collision Force. According to the collision hypothesis, Coriolis force, centrifugal force, and speed dependency can be ignored relative to the collision impulse force because the impact time is very short $[38,39]$. The integral in a very short period of time can be obtained:

$$
H^{*} \int_{t_{0}}^{t_{0}+\delta t} \ddot{q} d t=J^{* T} \varphi,
$$

where $\varphi=\int_{t_{0}}^{t_{0}+\delta t} F_{e} d t$ is impulse force of the end effector.
To make $\delta \dot{q}=\dot{q}_{t_{0}+\delta t}-\dot{q}_{t_{0}}$, it can be obtained that

$$
\begin{aligned}
H^{*} \delta \dot{q} & =J^{* T} \varphi, \\
\delta \dot{q} & =H^{*-1} J^{* T} \varphi .
\end{aligned}
$$

On account of kinematic relation (we assume that the system initial momentum and angular momentum are 0 ),

$$
\dot{x}_{e}=J^{*} \dot{q} .
$$

Derivate the equation and integrate the derivation in a very short period of time:

$$
\delta \dot{x}_{e}=J^{*} \delta \dot{q}=J^{*} H^{*-1} J^{* T} \varphi .
$$

As for a pulse vector, it equals the product of pulse amplitude and direction vector; that is, $\varphi=n \gamma$, where $n \in \mathbf{R}^{6}$ :

$$
\delta \dot{x}_{e}=D n \gamma,
$$

where $D=J^{*} H^{*-1} J^{* T}$ is the inertia matrix of the Jacobian matrix.

The collision process is divided into compression stage and recovery stage. The normal relative velocity is 0 on two objects' contact surface at the end of compression stage. We assume that the velocities before collision are $v_{e 1}$ and $v_{e 2}$ and the velocities at the final stage of compression are $\delta v_{e 1}$ and $\delta v_{e 2}$. Thus, it forms the following formula in the final stage of compression:

$$
n^{T}\left(v_{e 2}+\delta v_{e 2}-v_{e 1}-\delta v_{e 1}\right)=0
$$

where $n$ is normal vector of collision.

We assume that the impulse of object 1 in compression phase is $-n \gamma^{c}$. Thus the impulse of object 2 is $n \gamma^{c}$ :

$$
\begin{aligned}
& \delta v_{e 1}=-D_{1} n \gamma^{c}, \\
& \delta v_{e 2}=D_{2} n \gamma^{c} .
\end{aligned}
$$

We rewrite the equation of the collision pulse in the compression stage as

$$
\gamma^{c}=-\frac{n^{T}\left(v_{e 2}-v_{e 1}\right)}{n^{T}\left(D_{2}+D_{1}\right) n} .
$$

The Poisson model is assumed to be the collision between the manipulator and the target. Thus, the impulse of recovery phase is $\gamma^{r}=e \gamma^{c}$, where $0 \leq e \leq 1$ is coefficient of restitution and has contact with surfaces and materials:

$$
\gamma=-(1+e) \frac{N^{T}\left(v_{e 2}-v_{e 1}\right)}{N^{T}\left(D_{2}+D_{1}\right) N} .
$$

Thus, the factors (except for inherent factor) affected by visible pulse force are the direction $N$ of collision force, the Jacobi matrix of inertial object $D_{2}\left(D_{2}\right.$ is fixed value when the object is ready), relative velocity $\left(v_{e 2}-v_{e 1}\right)$, and joint angle function $D_{1}=J_{v e}^{*} H^{*-1} J_{v e}^{* T}$, where $J_{v e}^{*}$ is Jacobian matrix of generalized linear velocity and $H^{*-1}$ is generalized inertia matrix of manipulator.

Thus, the mapping relationship between the collision force and the terminal velocity and the joint angle is established. 
2.3. End Speed. According to the robotics, the robot Jacobian matrix $(J(q))$ is defined as the linear transformation from the velocity of end effector to that of joints, which can be seen as the transmission ratio of the velocity from the joint space to the Cartesian space [37]. That is,

$$
V=\dot{X}=J(q) \dot{q} .
$$

We assume that $d$ and $\delta$ refer to the differential displacement and displacement rotation of the end effector, respectively; $v$ and $w$ refer to linear velocity and angular velocity, respectively. As for the $i$ th joint, the direction of linear velocity it produced on the end effector is along $Z_{i}$ :

$$
\left[\begin{array}{l}
v \\
w
\end{array}\right]=\left[\begin{array}{c}
Z_{i} \\
0
\end{array}\right] \dot{q}_{i} .
$$

Therefore, the $i$ th column of the Jacobian matrix is obtained:

$$
J_{i}=\left[\begin{array}{c}
Z_{i} \\
0
\end{array}\right]
$$

As for the rotation of revolute joint, its angular velocity of end effector is

$$
w=Z_{i} q_{i}
$$

At the same time, the linear velocity of end effector is vector product:

$$
v=\left(Z_{i} \times{ }^{i} P_{n}^{0}\right) q_{i} .
$$

Therefore, the column $i$ of the Jacobian matrix is defined as

$$
J_{i}=\left[\begin{array}{c}
Z_{i} \times{ }^{i} P_{n}^{0} \\
Z_{i}
\end{array}\right]=\left[\begin{array}{c}
Z_{i} \times\left({ }_{i}^{0} R_{i}^{i} P_{n}\right) \\
Z_{i}
\end{array}\right],
$$

where ${ }^{i} P_{n}^{0}$ refers to the origin of the last frame in the frame $\{i\}$ relative to base frame.

The first three rows are on behalf of the transmission of the linear velocity $(v)$ and the last three rows represent the transmission rate of angular velocity $(w)$, while each column represents that the corresponding joint velocity has influence on linear velocity and angular velocity of end effector. Therefore, the Jacobian matrix $(J)$ of manipulator can be written in the form of block:

$$
\begin{aligned}
{\left[\begin{array}{c}
v \\
w
\end{array}\right] } & =\left[\begin{array}{llll}
J_{L 1} & J_{L 2} & \cdots & J_{L n} \\
J_{A 1} & J_{A 2} & \cdots & J_{A n}
\end{array}\right]\left[\begin{array}{c}
\dot{q}_{1} \\
\dot{q}_{2} \\
\vdots \\
\dot{q}_{n}
\end{array}\right], \\
\dot{X} & =\left[\begin{array}{lll}
v & w
\end{array}\right]^{T}, \\
J & =\left[\begin{array}{llll}
J_{L 1} & J_{L 2} & \cdots & J_{L n} \\
J_{A 1} & J_{A 2} & \cdots & J_{A n}
\end{array}\right], \\
\dot{q} & =\left[\begin{array}{llll}
\dot{q}_{1} & \dot{q}_{2} & \cdots & \dot{q}_{n}
\end{array}\right]^{T} .
\end{aligned}
$$

We can simplify the equation as

$$
\dot{X}=J \dot{q}
$$

The mapping relationship between the angular velocity of joint and velocity of end effector is established.

2.4. Energy Consumption. As the space mechanism is rather complex and the load of space vehicle is limited, it cannot carry more energy. As a result, the energy used by the arm is often limited. Energy used as an index of factors affecting the reliability of space mechanism is to reduce the energy consumption of the mechanical arm system and to meet the requirements of the mechanical arm using a long time. According to the calculation of the joint output power [40], we can put forward the mathematical relationship about energy as follows based on the solution of joint moment:

$$
f=\sum_{i=1}^{n} \int_{t_{0}}^{t_{f}}\left[\dot{q}_{i}(t) \tau_{i}(t)^{2}\right] d t .
$$

2.5. Friction and Wear. The friction and wear cannot be avoided on account of joint gap, gear occlusion, and flexibility [41]. The relationship between the controllable variables and friction can be obtained by the following friction model:

$$
\mu \dot{q}=J \ddot{q}-m g l \cos (q),
$$

where $\mu$ is friction coefficient, $J \ddot{q}$ is output torque, and $m g l \cos (q)$ is gravity torque.

\section{The Operational Reliability System Control Model}

The operational reliability system control model is illustrated in Figure 2. Three control levels, task planning, path planning, and motion control, are used to complete the basic control of the space manipulator. A feedback loop considering operational reliability factors (both the taskrelated factors and the cost-related factors) is added in the system to adjust the control process, delaying the mechanism performance degradation during the service period and improving the operational reliability. The fault diagnosis module, in the feedback loop, is used to assess the state of the system. When the fault section is in one of two states, the performance degradation state or functional failure state, the noise suppression method (bottom path of fault self-handling) or model reconstruction method (top path of fault self-handling) is used to adjust the control system, respectively.

3.1. Three-Layer Control Structures. The control system of the space manipulator is divided into three layers: task planning, path planning, and motion control, along the forward path of the control loop in Figure 2. A specified task is the input to the control system for the space manipulator, and the first module, task planning module, splits the task into stages; the first block, the Stage Division of Task block, divides the task into discrete stages. Then the Feasibility Analysis of Stage 


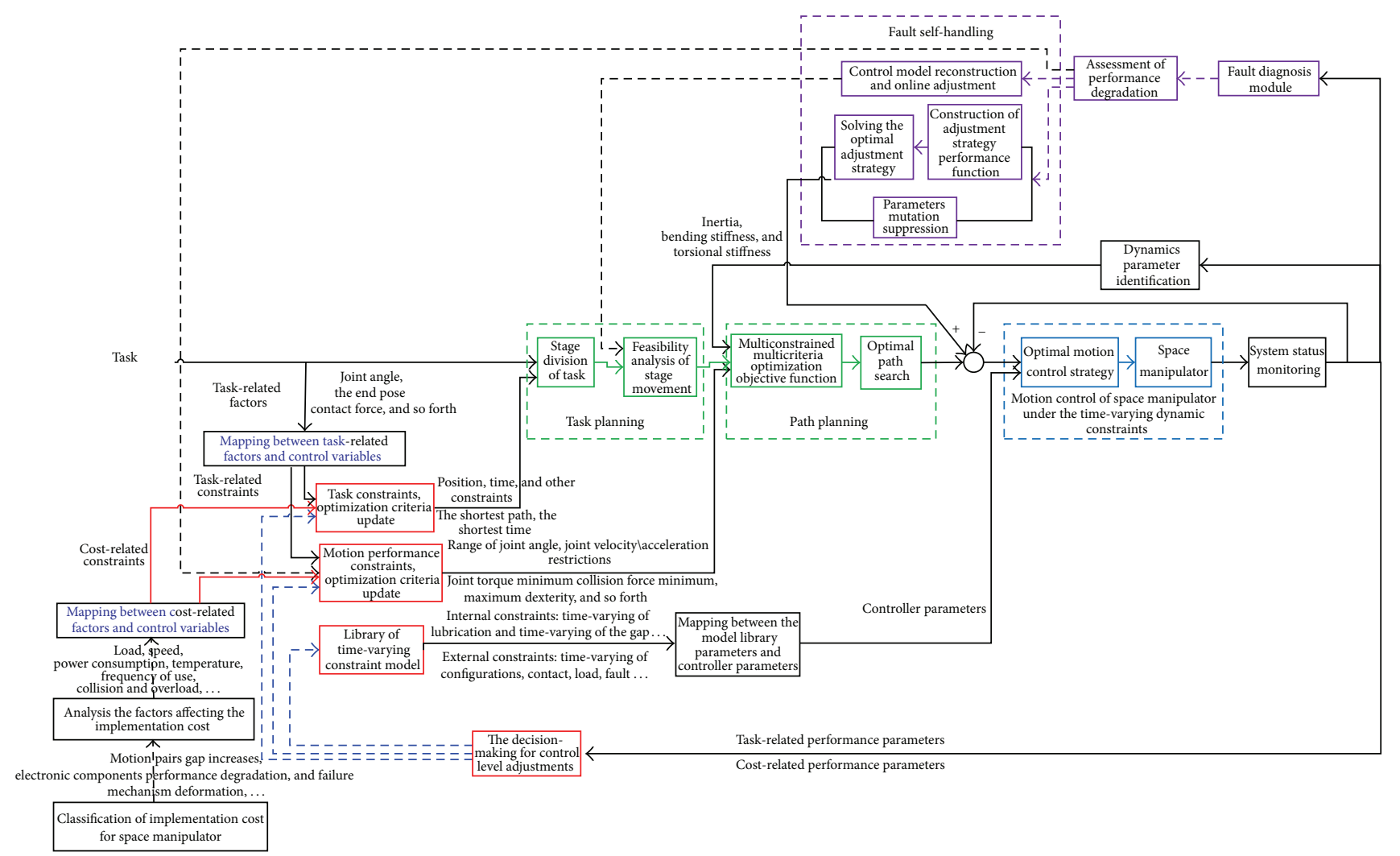

FIGURE 2: The operational reliability system control model of space manipulator.

Movement block divides each stage into a sequence of middle points. Path planning module plans the path between every two adjacent middle points and divides it into a sequence of joint angles in the time domain. According to the joint angle sequences obtained by the path planning module, motion control module controls the motors to make the joints rotate to the desired angles.

The detected value of the task-related performance parameters and cost-related performance parameters is the feedback information; taking the operational reliability factors into consideration, the system can autonomously decide which control level of system should be optimized and how to optimize it using the decision-making for control level adjustments module located in the feedback loop. The module makes decisions based on the following logic guidelines:

(1) The task-related parameters are in line with expectations, and cost-related parameters are reasonable: the system is normal. No adjustments to the constraints are imposed.

(2) The task-related parameters are in line with expectations, and cost-related parameters are unreasonable: path planning constraints should be adjusted.

(3) The task-related parameters are not in line with expectations, and cost-related parameters are reasonable: motion controller parameters should be adjusted.
(4) The task-related parameters are not in line with expectations, and cost-related parameters are unreasonable: potential fault and task planning should be adjusted.

Whether the task-related parameters are in line with expectations is determined in the decision-making for control level adjustments block as follows.

Denote by $I_{i}, \quad i=1, \ldots, n$, the value of the $c$ th task-related factor. For example, the joint angles $I$ can be measured and are the configuration of the end effector.

Let the error between the value of the task-related factors and that of the control target during each control period be denoted by

$$
\Delta I_{i j} \quad(i=1,2, \ldots n, j=1,2, \ldots m),
$$

where $\Delta I_{i j}$ is the error of the factors, $n$ is the number of factors, and $m$ is the control period.

Then the accumulated error of the task-related factors within $k$ control cycles is

$$
Q_{i t}=\sum_{j=t-k}^{t}\left(\Delta I_{i j}\right)^{2} \quad(i=1,2, \ldots n),
$$

where $Q_{i t}$ is the cumulative error of the task-related factors. $\Delta I_{i j}$ is the error of the factors. $n$ is the number of factors. $t$ is the current control cycle and $k$ is the integration length.

When $Q_{i t}$ is greater than a prescribed threshold value $Q_{i t}^{*}$, the task-related parameters do not meet the requirements; 
otherwise the task-related parameters meet the requirements. The threshold value $Q_{i t}^{*}$ is determined in accordance with task requirements and engineering experience.

Whether the cost-related parameters are reasonable is determined as follows. The factors of current, temperature, and so forth related with costs are expressed by $C_{i j}$ :

$$
C_{i j} \quad(i=1,2, \ldots n, j=1,2, \ldots m),
$$

where $C_{i j}$ means value of the factors related with costs and $n$ and $m$ are, respectively, the number of factors and control cycles.

A cumulative value of each factor related with costs in $k$ control cycles is taken:

$$
L_{i t}=\sum_{j=t-k}^{t} C_{i j} \quad(i=1,2, \ldots n),
$$

where cumulative error of factors related with costs is $L_{i t}$ and $C_{i j}$ is value of factors related with costs, with $n, t$, and $k$, respectively, expressing the number of factors, current control cycle, and the length of integration.

The cost-related parameters are not reasonable when $L_{i t}$ is bigger than the prescribed threshold value $L_{i t}^{*}$; otherwise they are reasonable. The threshold value $L_{i t}^{*}$ is determined according to engineering experience and experimental statistical data.

3.2. Adjustment Method of Control Levels. Based on the results obtained by the adjustment decision-making module of control level, different strategies are used to make adjustments or corrections for each control level. In task planning layer and path planning layer, the planning scheme is adjusted by changing constraints of control variables and optimization criteria, and the controller parameters are modified to improve performance in motion control layer.

The effect of flexible dynamics will be considered in path planning layer, which means that the operational reliability system control method will consider how to eliminate the influence of the flexible dynamics in the path planning. Based on it, new constraints in the path planning level are added up and optimal path planning trajectory is obtained, which can reduce or eliminate the vibration effect of robotic arm during the operation process.

In motion control layer, Library of Time-Varying Constraint Model includes internal constraints and external constraints. Internal constraints consist of time-varying of lubrication, time-varying of the gap, and so forth. External constraints consist of time-varying of configurations, timevarying of contact, time-varying of load, time-varying of fault, and so forth.

Based on the determined reliability factors, the corresponding relationships between the affecting factors of the operational reliability (such as gear friction torque, friction torque of bearings, and link bending deformation) and dynamics parameters of joint system (such as moment of inertia, stiffness, and internal friction torque) are analyzed, and Library of Time-Varying Constraint Model is built. Based on the above, with time-varying dynamics parameters as constraints, the joint movement control system framework of space manipulator is built. Online dynamics parameter identification method is used; then the feedforward compensation with the joint moment of inertia, stiffness and internal friction torque, and the feedback compensation using control deviation are realized. Finally the motion control method of operational reliability with time-varying dynamic constraints for space manipulator is achieved.

Exploring many possible strategies for this is the subject of current research efforts. The example later in the paper provides a possible implementation.

3.3. Adjustment of Control Level Introduced Influencing Factors of Operational Reliability. According to the mapping relationship between influencing factors of operational reliability and each control variable of manipulator in the multiple constraints and multivariable comprehensive optimization model, influencing factors of operational reliability under the condition of performing a task with high quality and low cost are selected, and scope of the corresponding control variables is obtained as their constraint range through the mapping relationship. This constraint is made as a new constraint of mission planning and path planning to replan the task or path.

3.4. Overview of Fault Self-Processing. Data from sensors and other monitoring modules are analyzed through the fault diagnosis module, judging whether there is fault and fault source. Aiming at fault source, influence function of operation ability under the condition of fault for space manipulator is established to evaluate performance attenuation.

If performance of the fault source attenuates, movement performance constraints and optimization criterion are changed according to the degree of attenuation, and noise suppression adjustment is implemented with adjustment amount as correction of motion control to ensure the maximum probability of completing a task under the fault condition.

If the fault diagnosis module detects a fault, fault selfhandling is implemented, including online adjustment and reconstruction of control model for manipulator and adjustment of restraining parameter mutation.

For redundant space manipulator, the freedom degrees of the joints are greater than the freedom degrees that the end pose defines. Within the joint space, there are multiple sets of feasible solution which can make each of the joints move from an initial position to a desired position while keeping the end actuator pose unchanged. Meanwhile, it is possible for redundant space manipulator to complete a given task while one or some joints fall into a complete joint failure. In this case, reconstruction and restraining of parameters mutation methods for space manipulator are required.

Model reconstruction includes kinematics and dynamics model reconstruction. Online adjustment includes the adjustment of control models and the operating parameters. The adjustment of control models includes adjustment of constraints, control objective, parameters mathematical relationship, and calculating the mathematical model again. The adjustment of motion parameters includes adjustment of the 
parameters mutation due to joint faults and model reconstruction. Restraining of parameters mutation is achieved by introducing compensation term to make the manipulator operate smoothly during its control model reconstruction and adjustment $[42,43]$.

After reconstruction and online adjustment of model for manipulator are implemented, the stage division achieved by task planning module is rejudged as feasible or not. If not, task constraints and optimization criteria are updated and task planning module is performed again.

\section{Simulation Experiment}

4.1. Experimental Scheme. The control method is further explained with a "no-load transfer task" of the space manipulator as an example. According to the multiple constraints and multivariable comprehensive optimization model of operational reliability, it is determined that the task-related factor for the "no-load transfer task" of the space manipulator is the terminal position accuracy. Joint wear is made as an example for the cost-related factor of the example, which only considers the effect of velocity on the wear.

Sensors are used to detect each joint angle of the manipulator for each control cycle. The measurement values are subtracted from the target joint angle in the path planning scheme to obtain the error of each joint angle and the error of the terminal position according to the forward kinematics solution of the manipulator. The sum of the squares of the terminal position error in $k$ control cycles is taken as only one example of a way to calculate the error of the taskrelated factor. When it is greater than the required threshold of the task, the effect of task execution is not consistent with expectations. For the rest of this example, we will assume that the task-related factors are less than the required threshold.

For the cost-related factors, according to the theory of tribology, wear of a kinematic pair inevitably requires increased power consumption, which causes the increase of working current for actuators. When the value of the current detected by a sensor is greater than a reasonable threshold, we take that as an indirect measure of the cost-related factor.

Based on the above information, the decision-making for control level adjustments block determines which control layer to adjust, if any:

(1) If the manipulator runs normally, none of the control levels needs to be adjusted.

(2) If a failure occurs, the task planning scheme needs to be adjusted. According to the fault self-handling, the failed joint is locked first, and then online adjustment and reconstruction of the control model for the manipulator are implemented by the control model reconstruction and online adjustment block, and then task feasibility evaluation is implemented to the model of the manipulator after reconstruction. If the reconstructed model cannot meet execution conditions of continuing the task, the task is replanned based on the new control model of the manipulator.

(3) If the cost-related factors are unreasonable, then path planning needs to be adjusted, and the joint angle range of error minimum and the joint angular velocity of wear rate
TABLE 1: The D-H parameters of the manipulator.

\begin{tabular}{lcccc}
\hline Link $i$ & $\alpha(\mathrm{rad})$ & $a(\mathrm{~mm})$ & $\theta(\mathrm{rad})$ & $d(\mathrm{~mm})$ \\
\hline 1 & 0 & 0 & 0 & 675 \\
2 & $-\pi / 2$ & 0 & $-\pi / 2$ & 110 \\
3 & $\pi / 2$ & 0 & 0 & 245 \\
\hline
\end{tabular}

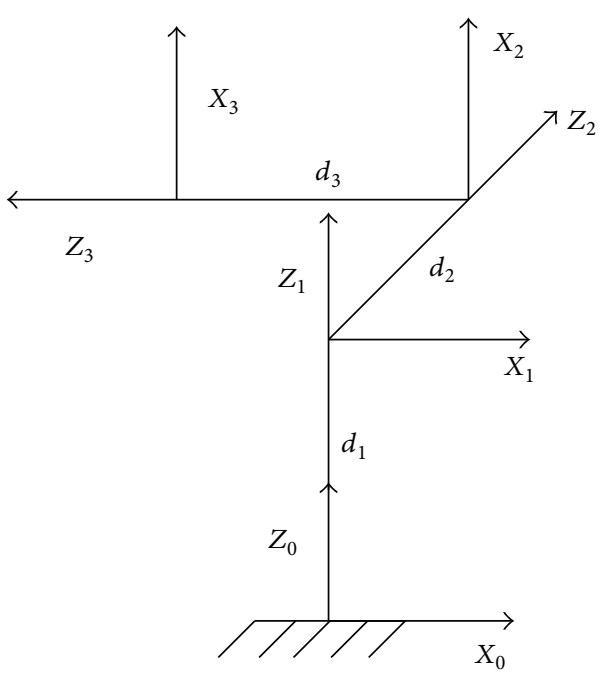

FIGURE 3: 3-DOF manipulator's D-H coordinate system.

minimum at the current time are determined according to the model of joint error and joint wear.

A manipulator model is built in Matlab. It has 3 DOF and each of them is rotary joint. The manipulator's D-H coordinate system is shown in Figure 3. The D-H parameters are shown in Table 1 , and joint variable is the joint angle $\theta$.

Based on the given kinematics parameters ( $\mathrm{D}-\mathrm{H}$ parameters) of manipulator, the kinematics model is established, and the mapping relationship between joint angle and end pose is obtained. By using the kinematics model and path planning algorithms, the path and joint angle sequence can be obtained for subsequent simulations. Using the joint angle sequence, combining with the wear model, the wear can be calculated while the manipulator performs a given path, and the followup simulations can be conducted.

The joint error model is the error distribution under different joint angles:

$$
e \sim F\left(p_{1}(\theta), p_{2}(\theta), \ldots, p_{n}(\theta)\right)
$$

where $e$ is joint error, $\theta$ is joint angle, and $F(\cdot)$ expresses the probability distribution of error with $p_{1}(\theta), p_{2}(\theta), \ldots, p_{n}(\theta)$ expressing parameters of the distribution $F(\cdot)$, which is obtained by experimental data statistics.

Model of joint error for simulation assumes that it obeys normal distribution:

$$
e \sim N\left(\mu, \sigma^{2}\right)
$$

where $\mu=0, \sigma=0.05 * 25 * f_{N}(\theta)+0.02$, and $f_{N}(\theta)=$ $(1 / \sqrt{2 \pi}) e^{-(\theta+0.5)^{2} / 2}$. 


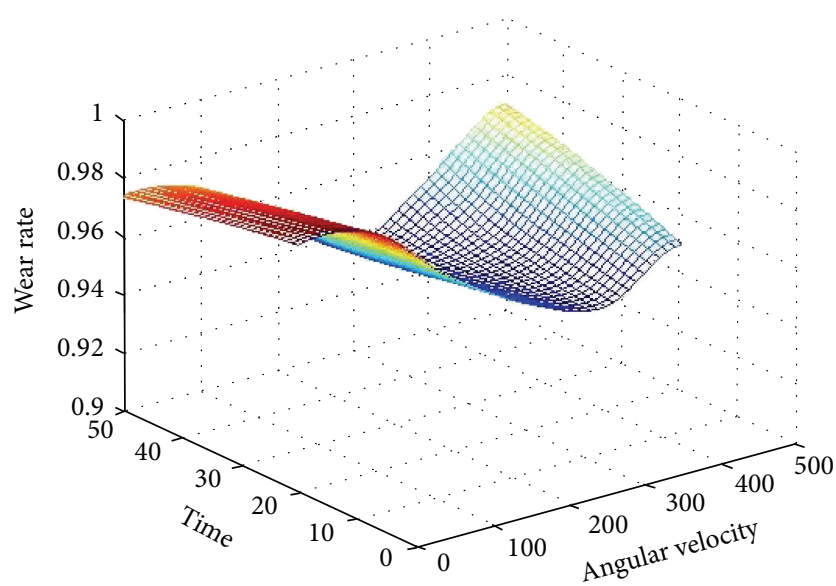

FIGURE 4: Schematic diagram of the time-varying wear model.

The joint system of manipulator is a highly nonlinear system. The friction and wear are important nonlinear factors that affect the control precision and the operational cost. The friction parameters change with time-varying factors (such as the position and the velocity of the joint) during the dynamic operational process of the manipulator.

The wear rate model should be derived by the analysis of wear theory or actual experiment test. No matter which method is adopted, the model should be a function related to multiple factors (one of the independent variables is time).

In this paper, a wear model is built based on the investigation and analysis of materials and relevant research reports [44-47]. For simplicity, the wear rate is only related to the change of time and joint angular velocity.

Model of joint wear is the wear rate under different joint angular velocity:

$$
\dot{w}=G(\dot{\theta}, t)
$$

where $\dot{w}, \dot{\theta}$, and $t$ are, respectively, wear rate, joint angular velocity, and service time of equipment and $G(\cdot)$ expresses the wear surface obtained by wear tests under different working conditions.

A time-varying wear model is introduced in the form of the wear rate distribution of different joint angular velocity and assuming time-varying wear model is as shown in Figure 4.

In the simulation experiment, we record the wear within the given service time. In order to calculate the wear, firstly, we traverse the service time of the manipulator. It performs the given task during each service phase and then the wear in each task control cycle is obtained according to the wear model. Then, the wear during the process of the entire task is obtained by integral approach. At last, we obtain the wear of the manipulator through the repeated simulation within the given service time.

When constraints of joint angle and joint angular velocity are determined, task and path planning for the manipulator are reevaluated. If the current planning scheme meets the constraint conditions, it continues to execute the task,

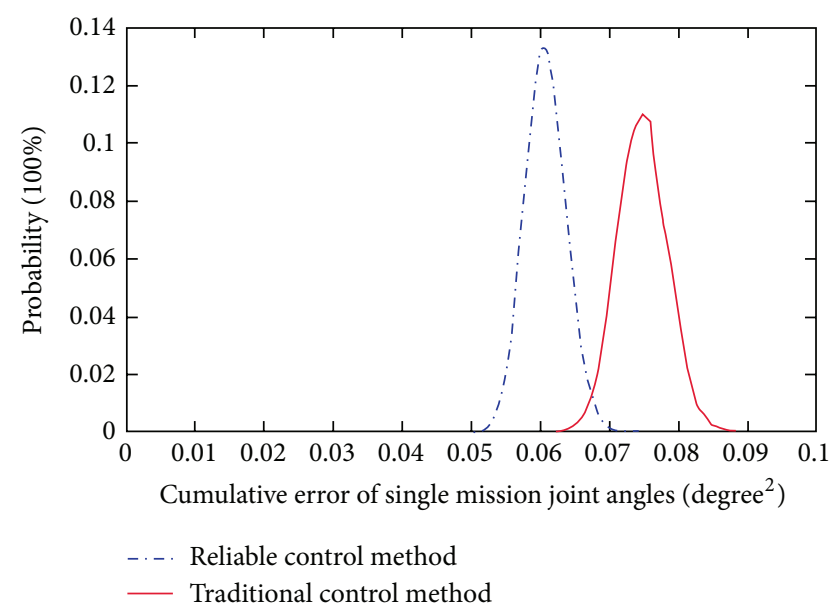

FIGURE 5: Comparisons of error distributions of different planning scheme.

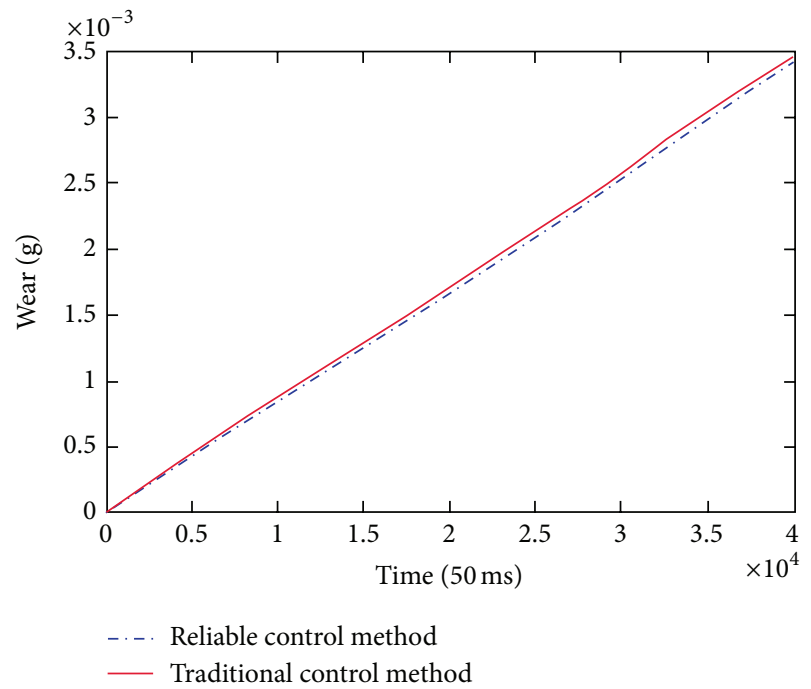

FIGURE 6: Comparisons of wear amount of executing the same task consistently.

otherwise replanning the scheme according to the new constraint conditions.

(4) If motion control needs to be adjusted, then parameters of the controller are updated according the mapping between the model library parameters and controller parameters block.

4.2. Results Analysis. As is shown in Figure 5, compared with the traditional control methods, error between planning scheme and the executing result falls in the range closer to zero by that of operational reliability with a greater probability of execution results meeting accuracy requirements of the task.

As is shown in Figure 6, the traditional control methods without considering the cost-related factors and that of operational reliability are used to execute the same task for a long time with a smaller amount of wear for manipulator by the control method of operational reliability. Thus, it can 
be inferred that, with other influencing factors of reliability changing identically and the same wear failure criteria, using the control method of operational reliability to control the manipulator has a longer expected service period.

\section{Conclusion}

From the aspect of research achievement, a control model of hierarchical system introducing operational reliability influencing factors is constructed from system level in this project, dividing the control process into three levels of task planning, path planning, and motion control. Actual state of operational reliability for space manipulator is real-time characterized and reflected through the detected or calculated value of operational reliability factors (terminal position, terminal velocity, collision force, energy consumption, friction and wear, etc.). It is made as a feedback to consider the mapping relationship between influencing factors of operational reliability and control variables comprehensively and the decision-making for control level adjustments module is used to decide adjustment strategy of three control levels autonomously.

Based on the obtained results of the decision-making for control level adjustments module and through the multicriteria task planning model under multiconstraint conditions, path planning model considering the effect of flexible dynamics, and motion control model under time-varying and dynamic constraints for space manipulator, the three control levels are influenced and adjusted by the operational reliability factors in the form of control variable constraints and different strategies are used to adjust or amend for each control level. In task planning and path planning level, planning scheme adjustment is influenced in the form of changing control variable constraints and optimization criteria, and performance is improved by modifying parameters of the controller in motion control level. A simulation example is designed based on the proposed control model of operational reliability, with a "no-load transfer task" of space manipulator as an example to further illustrate this method. In the experiment, joint wear is treated as the cost-related factor, only considering the effect of the control variable (joint angular velocity) on wear.

The result shows that, compared with the traditional control method executing the same task for a long time, the control method of using the operational reliability to control the manipulator has a smaller amount of wear, and this method has a longer expected service period under the same wear failure criteria. The research results can be well extended and applied to the development of aviation, navigation, and complex electromechanical products in other industries, which plays an important role in enhancing core competitiveness of the national science and technology and has very important scientific significance and practical value in engineering.

\section{Future Work}

(1) In the three control levels of the system control model, the introduced level and occasion of dynamics factors need to be considered. Task planning level is responsible for profile analysis of the given task, completing events and description of environmental timing sequence during the execution process of task. Due to the technical characteristics, it has not yet to consider the dynamic factors in task planning level. Therefore, dynamic factors are, respectively, introduced only in path planning and motion control.

In path planning, joint driven torque of the manipulator is limited under general conditions, so it should be reduced as much as possible under the premise to ensure the successful completion of the task. In order to solve this problem, the optimization objective function related to joint driving torque of space manipulator is established based on dynamic equations. Based on the local optimization methods (null space method, minimum joint torque method, etc.) or the global optimization methods (particle swarm optimization algorithm, etc.), objective function is optimized, namely, achieving the minimum joint torque. In motion control, flexibility, friction, and so on related with operational reliability are added to dynamic models in the design of the controller. Parameters of the above dynamic models are identified in the control process to adjust parameters of the controller in real time and optimize control effect.

(2) The system control model of operational reliability for space mechanism under fault mode should be further improved and perfected. Under fault conditions, it is a problem that should be solved in the subsequent research work, that is, how the control model of manipulator completes the fault self-handling and ensures compliance control of sudden fault and maximum probability of task completion. Data from monitoring module of sensors and so forth are analyzed to judge whether there is a fault and the fault source through the fault diagnosis module. Under fault conditions, scope of the degraded workspace for manipulator is determined through the Assessment of Performance Degradation module.

If the performance of fault source degrades, such as increase of the gap, being smaller than the maximum value of joint torque, the method of noise suppression is adopted and the noise is estimated by Bayesian method when noise interference aggravates. Then performance function of adjustment strategy is constructed to superpose a corrected value for motion control input to maximize the probability of completing the task successfully for the manipulator under fault conditions. If the fault source fails, such as the joint failure, locking the fault joint immediately, according to onorbit task requirements of space manipulator, and faulttolerant control optimization objectives based on operational reliability, basic control constraints are selected (including boundary constraints, kinematic constraints, and dynamic constraints), using the reconstruction method of control model to establish the fault-tolerant control model of space manipulator and suppressing parameter mutation at the same time. Because system model switching can cause the parameter mutation of the control model, the priority of suppressing mutation parameters is determined and the suppression method research considering global parameters mutation is developed for typical on-orbit tasks of space manipulator by using the principle of perturbation method and adding the vector of control compensation to derive suppression 
function of parameter mutation. Smooth switching from the original control model to fault-tolerant control model is realized based on online adjustment method of the model and suppression method of parameter mutation.

\section{Competing Interests}

The authors declare that there is no conflict of interests regarding the publication of this paper.

\section{Acknowledgments}

The authors would like to thank their colleagues from the Robotics Research Group for helpful discussions and comments on this paper. Meanwhile, the authors also would like to thank Professor J. William Goodwine at the University of Notre Dame for his valuable and helpful suggestions that led to significant improvements of this paper. This work is supported in part by Key Project of Chinese National Programs for Fundamental Research and Development (973 program) (no. 2013CB733000) and National Natural Science Foundation of China (no. 61573066).

\section{References}

[1] C. Toglia, F. Kennedy, and S. Dubowsky, "Cooperative control of modular space robots," Autonomous Robots, vol. 31, no. 2-3, pp. 209-221, 2011.

[2] G. Rekleitis and E. Papadopoulos, "On on-orbit passive object handling by cooperating space robotic servicers," in Proceedings of the IEEE/RSJ International Conference on Intelligent Robots and Systems1, pp. 25-30, San Francisco, Calif, USA, September 2011.

[3] S. Abiko and K. Yoshida, "Adaptive reaction control for space robotic applications with dynamic model uncertainty," Advanced Robotics, vol. 24, no. 8-9, pp. 1099-1126, 2010.

[4] T.-C. Nguyen-Huynh and I. Sharf, "Adaptive reactionless motion for space manipulator when capturing an unknown tumbling target," in Proceedings of the IEEE International Conference on Robotics and Automation (ICRA '11), pp. 4202-4207, IEEE, Shanghai, China, May 2011.

[5] M. D. Carpenter and M. A. Peck, "Reducing base reactions with gyroscopic actuation of space-robotic systems," IEEE Transactions on Robotics, vol. 25, no. 6, pp. 1262-1270, 2009.

[6] A. E. Jimenez-Cano, J. Martin, G. Heredia, A. Ollero, and R. Cano, "Control of an aerial robot with multi-link arm for assembly tasks," in Proceedings of the IEEE International Conference on Robotics and Automation (ICRA '13), pp. 4916-4921, Karlsruhe, Germany, May 2013.

[7] L. Marconi, R. Naldi, and L. Gentili, "Modelling and control of a flying robot interacting with the environment," Automatica, vol. 47, no. 12, pp. 2571-2583, 2011.

[8] Y.-C. Liu and N. Chopra, "Control of semi-autonomous teleoperation system with time delays," Automatica, vol. 49, no. 6, pp. 1553-1565, 2013.

[9] A. Keemink, M. Fumagalli, S. Stramigioli, and R. Carloni, "Mechanical design of a manipulation system for unmanned aerial vehicles," in Proceedings of the IEEE International Conference on Robotics and Automation (ICRA '12), pp. 3147-3152, St Paul, Minn, USA, May 2012.
[10] P. Acquatella, "Development of automation \& robotics in space exploration," in Proceedings of the AIAA SPACE Conference \& Exposition, pp. 1-7, Pasadena, Calif, USA, June 2009.

[11] C. Kaiser, P. Rank, and R. Krenn, "Simulation of the docking phase for the SMART-OLEV satellite servicing mission," in Proceedings of the 9th International Symposium on Artificial Intelligence, Robotics and Automation in Space, pp. 26-29, Los Angeles, Calif, USA, Februar 2008.

[12] S. Krut, M. Benoit, E. Dombre, and F. Pierrot, "MoonWalker, a lower limb exoskeleton able to sustain bodyweight using a passive force balancer," in Proceedings of the IEEE International Conference on Robotics and Automation (ICRA '10), pp. 22152220, May 2010.

[13] E. Zio, "Reliability engineering: old problems and new challenges," Reliability Engineering and System Safety, vol. 94, no. 2, pp. 125-141, 2009.

[14] G. J. Savage and Y. Kap Son, “The set-theory method for systems reliability of structures with degrading components," Reliability Engineering and System Safety, vol. 96, no. 1, pp. 108-116, 2011.

[15] W. Robert Brown and A. Galip Ulsoy, "A passive-assist design approach for improved reliability and efficiency of robot arms," in Proceedings of the IEEE International Conference on Robotics and Automation (ICRA '11), pp. 4927-4934, Shanghai, China, May 2011.

[16] E. Tian, D. Yue, and C. Peng, "Reliable control for networked control systems with probabilistic sensors and actuators faults," IET Control Theory \& Applications, vol. 4, no. 8, pp. 1478-1488, 2010.

[17] V. Chandra, Z. Huang, and R. Kumar, "Automated control synthesis for an assembly line using discrete event system control theory," IEEE Transactions on Systems, Man and Cybernetics Part C: Applications and Reviews, vol. 33, no. 2, pp. 284-289, 2003.

[18] Z. Liu, C. Chen, Y. Zhang, and C. L. P. Chen, "Adaptive neural control for dual-arm coordination of humanoid robot with unknown nonlinearities in output mechanism," IEEE Transactions on Cybernetics, vol. 45, no. 3, pp. 521-532, 2015.

[19] F. Zheng and M. Chen, "New conceptual structure design for affordable space large deployable antenna," IEEE Transactions on Antennas and Propagation, vol. 63, no. 4, pp. 1351-1358, 2015.

[20] D. Hirano, Y. Fujii, S. Abiko, R. Lampariello, K. Nagaoka, and K. Yoshida, "Simultaneous control for end-point motion and vibration suppression of a space robot based on simple dynamic model," in Proceedings of the IEEE International Conference on Robotics and Automation (ICRA '14), pp. 6631-6637, IEEE, Hong Kong, June 2014.

[21] G. Antonelli, "Stability analysis for prioritized closed-loop inverse kinematic algorithms for redundant robotic systems," IEEE Transactions on Robotics, vol. 25, no. 5, pp. 985-994, 2009.

[22] F. Aghili, "Adaptive control of manipulators forming closed kinematic chain with inaccurate kinematic model," IEEE/ASME Transactions on Mechatronics, vol. 18, no. 5, pp. 1544-1554, 2013.

[23] M. Otte and N. Correll, "C-FOREST: parallel shortest path planning with superlinear speedup," IEEE Transactions on Robotics, vol. 29, no. 3, pp. 798-806, 2013.

[24] L. Wang, T. Chai, and C. Yang, "Neural-network-based contouring control for robotic manipulators in operational space," IEEE Transactions on Control Systems Technology, vol. 20, no. 4, pp. 1073-1080, 2012.

[25] S. K. Pradhan and B. Subudhi, "Real-time adaptive control of a flexible manipulator using reinforcement learning," IEEE 
Transactions on Automation Science and Engineering, vol. 9, no. 2, pp. 237-249, 2012.

[26] M. Galicki, "Control of mobile manipulators in a task space," IEEE Transactions on Automatic Control, vol. 57, no. 11, pp. 2962-2967, 2012.

[27] J. Su and W. Xie, "Motion planning and coordination for robot systems based on representation space," IEEE Transactions on Systems, Man, and Cybernetics, Part B: Cybernetics, vol. 41, no. 1, pp. 248-259, 2011.

[28] M. Stilman, "Global manipulation planning in robot joint space with task constraints," IEEE Transactions on Robotics, vol. 26, no. 3, pp. 576-584, 2010.

[29] X. Ding and C. Fang, "A novel method of motion planning for an anthropomorphic arm based on movement primitives," IEEE/ASME Transactions on Mechatronics, vol. 18, no. 2, pp. 624-636, 2013.

[30] C.-F. Hsu, "Self-organizing adaptive fuzzy neural control for a class of nonlinear systems," IEEE Transactions on Neural Network, vol. 18, no. 4, pp. 1232-1241, 2007.

[31] C.-K. Lin, "Nonsingular terminal sliding mode control of robot manipulators using fuzzy wavelet networks," IEEE Transactions on Fuzzy Systems, vol. 14, no. 6, pp. 849-859, 2006.

[32] S. V. Shah, I. Sharf, and A. K. Misra, "Reactionless path planning strategies for capture of tumbling objects in space using a dualarm robotic system," in Proceedings of the AIAA Guidance, Navigation, and Control Conference, pp. 191-202, Boston, Mass, USA, August 2013.

[33] K. Nagai, Y. Dake, Y. Shiigi, R. C. V. Loureiro, and W. S. Harwin, "Design of redundant drive joints with double actuation using springs in the second actuator to avoid excessive active torques," in Proceedings of the IEEE International Conference on Robotics and Automation (ICRA '10), pp. 805-812, IEEE, Anchorage, Alaska, USA, May 2010.

[34] Z. Lian and E. del Castillo, "Setup adjustment under unknown process parameters and fixed adjustment cost," Journal of Statistical Planning and Inference, vol. 136, no. 3, pp. 1039-1060, 2006.

[35] Z. P. Mourelatos and J. Zhou, "Reliability estimation and design with insufficient data based on possibility theory," AIAA Journal, vol. 43, no. 8, pp. 1696-1705, 2005.

[36] X. Gao, Y. F. Wang, H. X. Sun et al., "Research on construction method of operational reliability control model for space manipulator based on particle filter," Mathematical Problems in Engineering, vol. 2015, Article ID 369747, 11 pages, 2015.

[37] J. Denavit and R. S. Hartenberg, "A kinematic notation for lower-pair mechanisms based on matrices," Journal of Applied Mechanics, vol. 22, pp. 215-221, 1955.

[38] K. Yoshida, "Space robot dynamics and control: to orbit, from orbit, and future," Robotics Research, vol. 9, pp. 449-456, 1999.

[39] O. Khatib, "A unified approach for motion and force control of robot manipulators: the operational space formulation," IEEE Journal of Robotics And Automation, vol. 3, no. 1, pp. 43-53, 1987.

[40] G. Field and Y. Stepanenko, "Iterative dynamic programming: an approach to minimum energy trajectory planning for robotic manipulators," in Proceedings of the 13th IEEE International Conference on Robotics and Automation, vol. 3, pp. 2755-2760, Minneapolis, Minn, USA, April 1996.

[41] T.-J. Tarn, A. K. Bejczy, X. Yun, and Z. Li, "Effect of motor dynamics on nonlinear feedback robot arm control," IEEE Transactions on Robotics and Automation, vol. 7, no. 1, pp. 114$122,1991$.
[42] Q. Jia, T. Li, G. Chen, H. Sun, and J. Zhang, “Trajectory optimization for velocity jumps reduction considering the unexpectedness characteristics of space manipulator jointlocked failure," International Journal of Aerospace Engineering, vol. 2016, Article ID 7819540, 14 pages, 2016.

[43] Q. Jia, J. Zhang, G. Chen, H. Sun, and T. Li, "Trajectory optimization of modular robot based on the fault-tolerant performance functions," in Proceedings of the IEEE 10th Conference on Industrial Electronics and Applications (ICIEA '15), pp. 580585, Auckland, New Zealand, June 2015.

[44] P. Tomei, "Robust adaptive friction compensation for tracking control of robot manipulators," IEEE Transactions on Automatic Control, vol. 45, no. 11, pp. 2164-2169, 2000.

[45] C. Canudas de Wit, H. Olsson, K. J. Aström, and P. Lischinsky, "A new model for control of systems with friction," IEEE Transactions on Control Systems Technology, vol. 40, no. 3, pp. 419-425, 1995.

[46] Y. Zhu and P. R. Pagilla, "Static and dynamic friction compensation in trajectory tracking control of robots," in Proceedings of the IEEE International Conference on Robotics and Automation (ICRA '02), pp. 2644-2649, IEEE, Washington, DC, USA, May 2002.

[47] M. Ruderman, F. Hoffmann, and T. Bertram, "Modeling and identification of elastic robot joints with hysteresis and backlash," IEEE Transactions on Industrial Electronics, vol. 56, no. 10, pp. 3840-3847, 2009. 


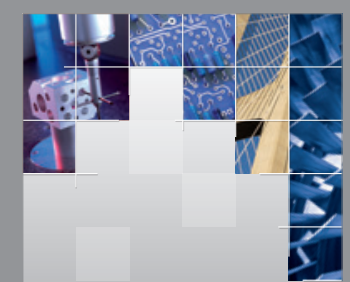

\section{Enfincering}
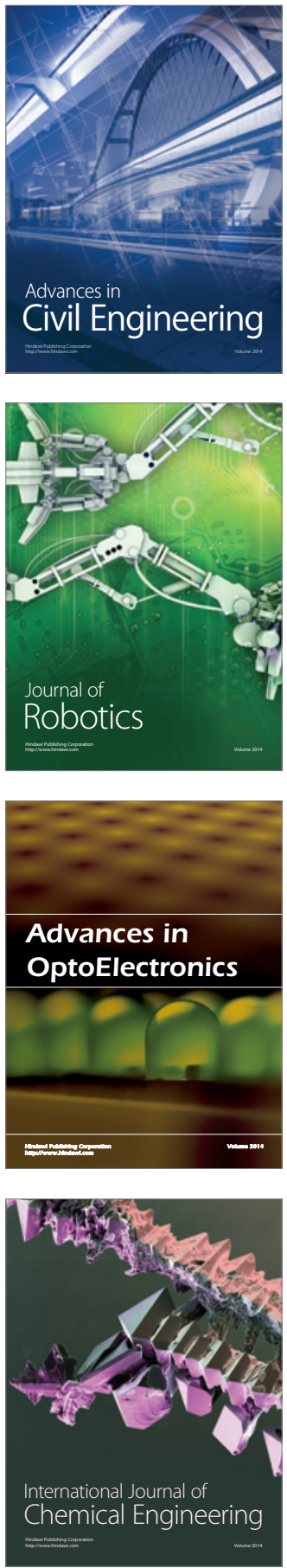

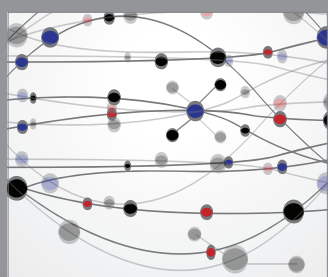

The Scientific World Journal

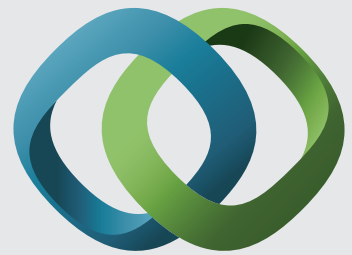

\section{Hindawi}

Submit your manuscripts at

http://www.hindawi.com
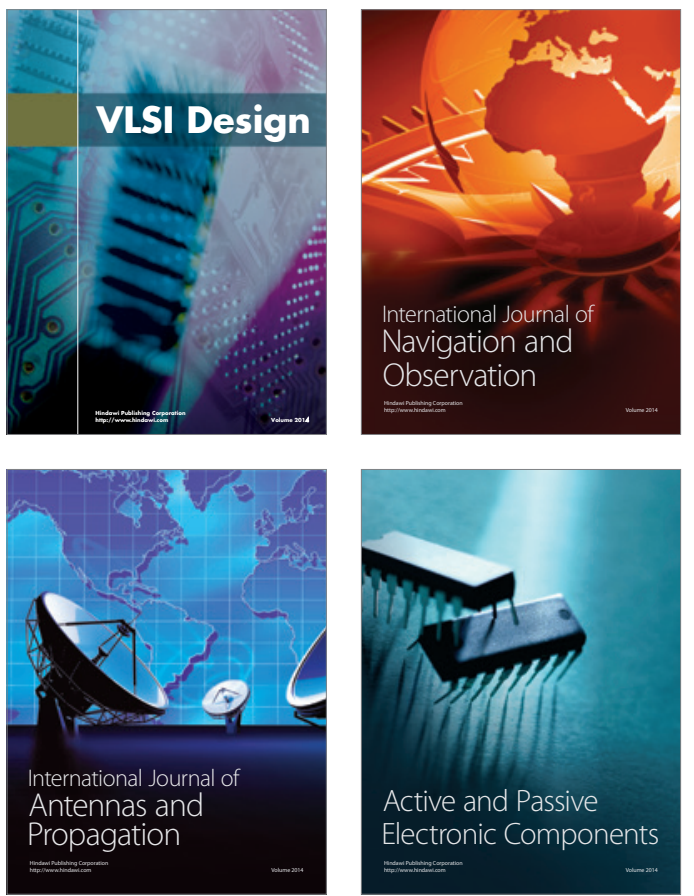
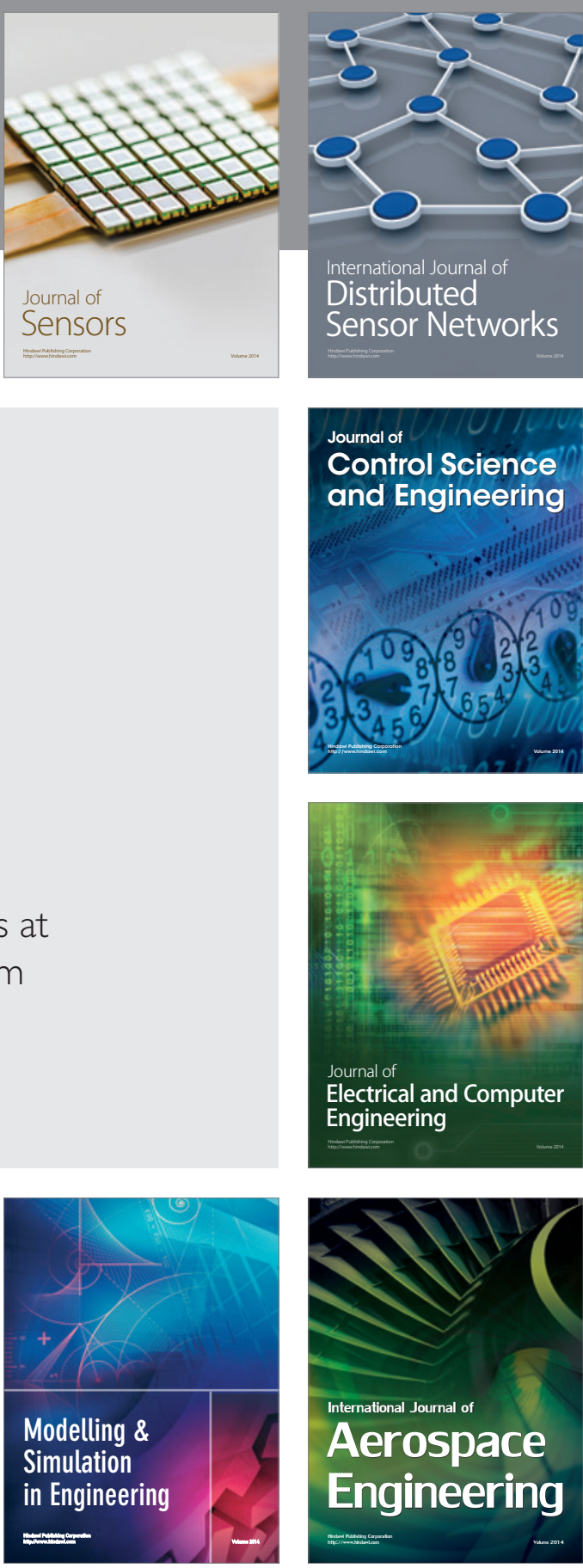

International Journal of

Distributed

Sensor Networks

Journal of

Control Science

and Engineering
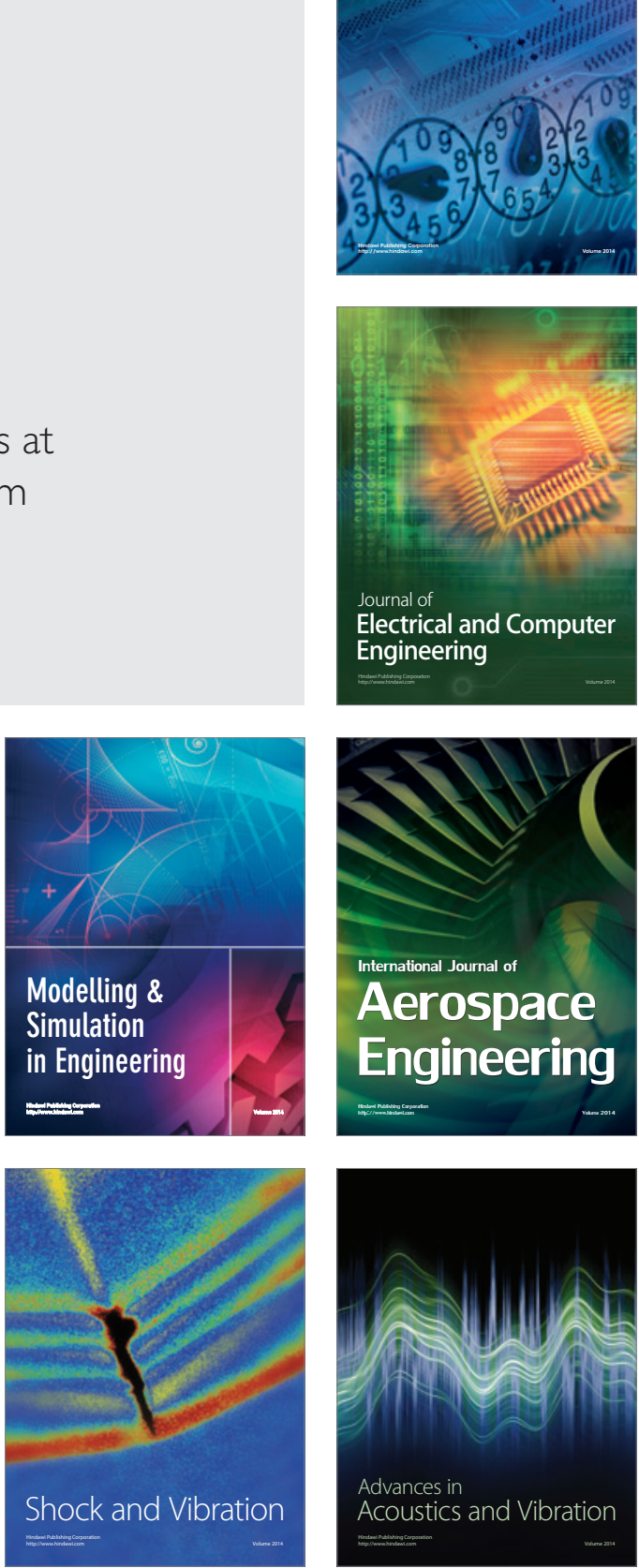